\title{
Validação de conteúdo de instrumento para verificar o tempo de uso de tela na infância
}

\author{
Content validation of an instrument to verify the screentime in childhood \\ Validación del contenido del instrumento para verificar el tiempo de uso de la pantalla en la \\ infancia
}

Recebido: 04/12/2021 | Revisado: 10/12/2021 | Aceito: 14/12/2021 | Publicado: 20/12/2021

Leticia Rodrigues Alves Bispo

ORCID: https://orcid.org/0000-0001-5936-1968 Universidade de São Paulo, Brasil

E-mail: leticia.bispo@usp.br

Matheus Francoy Alpes

ORCID: https://orcid.org/0000-0001-9617-7668

Universidade de São Paulo, Brasil

E-mail: matheus.alpes@usp.br

Patrícia Pupin Mandrá

ORCID: https://orcid.org/0000-0002-2926-0354

Universidade de São Paulo, Brasil

E-mail: ppmandra@fmrp.usp.br

\begin{abstract}
Resumo
Objetivos: Desenvolver e validar o conteúdo do instrumento "Tempo de Tela" destinado a pais e/ou responsáveis por crianças no período pré-escolar. Metodologia: Foram selecionados 5 especialistas na área de interesse para julgar o instrumento através de um formulário com 12 questões dicotômicas. A análise descritiva foi realizada calculando a frequência simples e para as respostas dissertativas foi realizada a análise de conteúdo. Resultados: $100 \%$ dos juízes julgaram a abordagem ao tema, direcionamento ao conteúdo, clareza, coerência e coesão, distribuição das questões e aspecto visual como itens adequados. As modificações consideradas foram: adicionar uma questão (40\%) e propostas de mudanças estéticas (40\%). Dentre as sugestões de adição de questões: grau de escolaridade da criança; se estuda em escola pública ou privada; nível socioeconômico dos pais; percepção dos pais em relação ao tempo de tela. Em relação às mudanças estéticas foram citadas: maior espaço para questões abertas; maior espaço entre uma questão e outra; alinhamento ao meio das frases dos tópicos; utilizar escala Likert; manterá mesma questão em uma única página. Conclusão: $\mathrm{O}$ instrumento foi submetido às etapas de validação de conteúdo preconizadas em literatura $\mathrm{e}$ preenche os requisitos quanto à sua face e conteúdo.
\end{abstract}

Palavras-chave: Fonoaudiologia; Linguagem infantil; Família; Tempo de Tela; Estudos de Validação.

\begin{abstract}
Purpose: To develop and validate the content of the "Tempo de Tela" instrument for parents and/or guardians of children in the preschool period. Methods: 5 experts in the area of interest were selected to judge the instrument to be validated through a form with 12 dichotomous questions. The descriptive analysis was performed by calculating the simple frequency and content analysis was performed for the essay answers. Results: $100 \%$ of the judges judged the approach to the theme, direction to the content, clarity, coherence and cohesion, distribution of the questions and visual aspect as appropriate items. The changes considered were: adding a question (40\%) and proposals for aesthetic changes (40\%). Among the suggestions for adding questions are: The child's educational level; if you study in a public or private school; parents' socioeconomic level; parents' perception of screen time. Regarding aesthetic changes, the following were mentioned: greater space for open questions; greater space between one question and another; alignment in the middle of the topic sentences; use Likert scale; keep the same question on a single page. Conclusion: The instrument was subjected to the content validation steps recommended in the literature and fulfills the requirements regarding its face and content.
\end{abstract}

Keywords: Speech, Language and Hearing Sciences; Child Language; Family; Screen Time; Validation Study.

\section{Resumen}

Objetivos: Desarrollar y validar el contenido del instrumento "Tiempo de Pantalla" para padres y / o tutores de niños en el período preescolar. Metodología: se seleccionaron 5 especialistas en el área de interés para juzgar el instrumento a través de un formulario con 12 preguntas dicotómicas. El análisis descriptivo se realizó mediante el cálculo de la 
frecuencia simple y para las respuestas del ensayo se realizó un análisis de contenido. Resultados: 100\% de los jueces juzgaron el enfoque del tema, la dirección del contenido, la claridad, la coherencia y cohesión, la distribución de las preguntas y la apariencia visual como ítems adecuados. Las modificaciones consideradas fueron: agregar una pregunta (40\%) y propuestas de cambios estéticos (40\%). Entre las sugerencias para agregar preguntas: nivel de educación del niño; si estudias en una escuela pública o privada; nivel socioeconómico de los padres; percepción de los padres del tiempo frente a la pantalla. En cuanto a los cambios estéticos, se mencionaron los siguientes: mayor espacio para preguntas abiertas; mayor espacio entre una pregunta y otra; alineación de temas a mitad de la oración; utilizar escala Likert; mantenga la misma pregunta en una sola página. Conclusión: El instrumento fue sometido a los pasos de validación de contenido recomendados en la literatura y cumple con los requisitos en cuanto a su cara y contenido.

Palabras clave: Fonoaudiología; Lenguaje infantil; Familia; Tiempo de Pantalla; Estudio de Validación.

\section{Introdução}

A exposição aos dispositivos eletrônicos se dá cada vez mais cedo. Essa questão tem despertado o interesse de pais, educadores e profissionais de saúde que fazem parte e acompanham o desenvolvimento infantil e são capazes de perceber as influências dessa exposição tão precoce, sejam elas positivas ou negativas em aspectos físicos, cognitivos, emocionais e de comunicação (Maziero et al., 2016).

A relação das crianças com as mídias e seu simbolismo cultural constitui um dos processos mais intensos que alcançam o ser humano nas sociedades modernas (De Paula \& Pereira, 2018). As relações interpessoais têm sido substituídas pelo universo atrás das telas que se tornou muito mais atrativa para a geração atual. (Reis \& Ziegler, 2016).

Embora crianças pequenas estejam mais suscetíveis à exposição a tecnologias do que no passado, é possível observar que elas aprendem mais a partir de ações ao vivo do que no equivalente em mídias digitais. Estudos têm apontado que a exposição à mídia antes dos 2 anos de idade pode causar mais efeitos negativos do que positivos. Acima desta idade, é possível que haja um benefício em aspectos de aprendizagem, porém, por serem estudos realizados majoritariamente em relação à exposição à televisores, faz-se necessária maior investigação a respeito de aplicativos de aparelhos móveis como celulares e tablets (Pereira, 2017).

A Sociedade Brasileira de Pediatria (SBP) recomenda que o tempo de uso diário "seja limitado e proporcional às idades e às etapas do desenvolvimento cerebral-mental-cognitivo-psicossocial das crianças e adolescentes" (SBP, 2016). Seguindo a mesma linha, a Organização Mundial da Saúde (OMS) recomenda que, até dois anos de idade, a exposição a telas não deve existir e que, a partir dos dois anos, o tempo não deve ultrapassar uma hora por dia (OMS, 2016).

O conhecimento e crenças de pais sobre o desenvolvimento infantil pode ser denominado como cognição parental. Idade, sexo, número de filhos, nível educacional e tempo diário de convivência são variáveis que influenciam o entendimento de responsáveis acerca do processo de aquisição e desenvolvimento infantil global e de habilidades e funções associadas, dentre elas, a linguagem (Macarini et al., 2010).

Sendo assim, o objetivo deste estudo foi desenvolver e validar o conteúdo do instrumento "Tempo de Tela" destinado a pais e/ou responsáveis por crianças no período pré-escolar.

\section{Metodologia}

\section{Aspectos éticos}

O estudo do tipo descritivo com abordagem quali-quantitativo foi submetido e aprovado pelo Hospital das Clínicas da Faculdade de Medicina de Ribeirão Preto (HCFMRP-USP) sob n ${ }^{\circ}$ 2.436.086/2018. Os juízes assinaram o Termo de Consentimento Livre e Esclarecido (TCLE), conforme Resolução 466/2012.

\section{Critérios de seleção de participantes}

O universo amostral da pesquisa foi composto por fonoaudiólogos com atuação na área de linguagem infantil, selecionados a partir de busca na Plataforma Lattes do site do Conselho Nacional de Desenvolvimento Científico e 


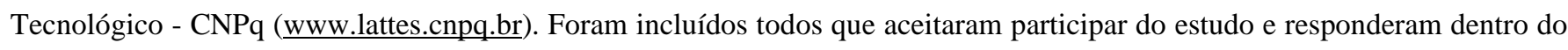
prazo estabelecido.

Os critérios de inclusão foram: ser fonoaudiólogo e realizar atendimentos na área de linguagem infantil; e ter sua participação no estudo devidamente autorizada mediante aceite através assinatura do Termo de Consentimento Livre e Esclarecido (TCLE), conforme Resolução CNS 466/2012. Não houve critérios de exclusão.

\section{Instrumento de coleta}

Um instrumento com 12 perguntas fechadas do tipo questionário com respostas dicotômicas (sim/não) foi elaborado considerando a literatura sobre pesquisas de validação (Alexandre et al., 2020; Alpes, 2018), para que os juízes pudessem avaliar o instrumento proposto. O valor de significância empregado foi de $0,8 \%$. Ao lado, o juiz poderia descrever suas sugestões e/ou comentários em um espaço designado para isto e a ficha de preenchimento para avaliação do questionário. O cabeçalho continha a instrução, explicando que o juiz deveria ler primeiro o instrumento e depois preencher a ficha.

\section{Procedimentos de validação}

O questionário para verificar o tempo de tela (TT) continha vinte e cinco questões, divididas em duas partes: I) identificação do responsável e da criança e II) descrição do uso dos aparatos eletrônicos, bem como fatores associados a este no cotidiano da família. Após aplicação inicial, com familiares de crianças de 2:0 a 5:11, foram realizadas modificações e a ampliação do instrumento que passou a conter quarenta questões, divididas em três partes: I) dados pessoais do respondente e da criança; II) uso dos aparelhos eletrônicos; III) marcos do desenvolvimento da infância e linguagem, e uma questão aberta para caso haja alguma outra consideração sobre os assuntos tratados no instrumento.

Os fonoaudiólogos foram convidados por meio de e-mail pessoal e os que aceitaram receberam por email um formulário do Google contendo o TCLE e o instrumento de validação, e em anexo o TT.

\section{Resultados}

Participaram como juízes cinco fonoaudiólogas com experiência clínica e teórica em linguagem infantil. As tabelas abaixo apresentam uma síntese do perfil sociodemográfica dos juízes participantes (sexo, ano de formação, titulação máxima e área de atuação).

Quanto ao sexo, os juízes participantes eram do sexo feminino (100\%), conforme descrito na Tabela 1. Este fato se dá por se tratar de uma profissão majoritariamente feminina no Estado de São Paulo: 97,9\% (DIEESE, 2018).

Tabela 1 - Caracterização dos juízes de acordo com sexo.

\begin{tabular}{ccc}
\hline Sexo & Número de especialistas & Frequência \\
Relativa (\%) & $1-0 \%$ \\
Feminino & 5 & $0 \%$ \\
Masculino & 0 & $100 \%$ \\
TOTAL & 5 & \\
\hline
\end{tabular}

Fonte: Bispo et al. (2021)

O tempo de formação variou entre 4 e 17 anos, sendo um (20\%) graduado entre 2003-2006, dois (40\%) entre 2007 2010, um (20\%) entre 2011-2013 e um (20\%) entre 2014-2017, conforme apresentado na Tabela 2.

Tabela 2 - Caracterização dos juízes de acordo com o ano de formação. 
Research, Society and Development, v. 10, n. 17, e97101724357, 2021

(CC BY 4.0) | ISSN 2525-3409 | DOI: http://dx.doi.org/10.33448/rsd-v10i17.24357

\begin{tabular}{ccc}
\hline Ano de Formação & Número de juízes & $\begin{array}{c}\text { Frequência } \\
\text { relativa }(\%)\end{array}$ \\
$2003-2006$ & 1 & $20 \%$ \\
$2007-2010$ & 2 & $40 \%$ \\
$2011-2013$ & 1 & $20 \%$ \\
$2014-2017$ & 1 & $20 \%$ \\
TOTAL & 5 & $100 \%$ \\
\hline
\end{tabular}

Fonte: Bispo et al. (2021)

A titulação máxima dos juízes foi de 2 (40\%) a nível de Mestrado e 3 (60\%) de Doutorado (Tabela 3). O estudo foi realizado com experts na área, o que justifica a alta titulação da maioria das profissionais.

Tabela 3 - Caraterização dos juízes de acordo com sua titulação máxima.

\begin{tabular}{ccc}
\hline Titulação máxima & Número de especialistas & Frequência relativa (\%) \\
Mestrado & 2 & $40 \%$ \\
Doutorado & 3 & $60 \%$ \\
TOTAL & 5 & $100 \%$ \\
\hline
\end{tabular}

Fonte: Bispo et al. (2021)

Quanto à área de atuação, três (60\%) dos juízes atuavam em assistência clínica, um (20\%) em docência e um (20\% em pesquisa (Tabela 4). 
Tabela 4 - Caracterização dos juízes de acordo com sua área de atuação.

\begin{tabular}{ccc}
\hline Área de atuação & Número de especialistas & Frequência relativa (\%) \\
Assistência Clínica & 3 & $60 \%$ \\
Docência & 1 & $20 \%$ \\
Pesquisa & 1 & $20 \%$ \\
TOTAL & 5 & $100 \%$ \\
\hline
\end{tabular}

Fonte: Bispo et al. (2021)

A Tabela 5 registra os quesitos que foram avaliados no questionário através do formulário e suas respectivas respostas.

Tabela 5 - Avaliação dos Especialistas em Linguagem infantil.

\begin{tabular}{|c|c|c|c|c|c|c|c|}
\hline Quesito & $\mathrm{J} 1$ & $\mathrm{~J} 2$ & $\mathrm{~J} 3$ & $\mathrm{~J} 4$ & J5 & $\begin{array}{l}\text { Frequência } \\
\text { simples (\%) }\end{array}$ & Resultado \\
\hline Abordagem ao Tema & SIM & SIM & SIM & SIM & SIM & $100 \%$ & $\begin{array}{c}\text { SEM } \\
\text { ALTERAÇÕES }\end{array}$ \\
\hline $\begin{array}{l}\text { Direcionamento ao } \\
\text { público-alvo }\end{array}$ & SIM & SIM & SIM & SIM & SIM & $100 \%$ & $\begin{array}{c}\text { SEM } \\
\text { ALTERAÇÕES }\end{array}$ \\
\hline Instruções & SIM & SIM & SIM & NÃO & SIM & $80 \%$ & $\begin{array}{c}\text { SEM } \\
\text { ALTERAÇÕES }\end{array}$ \\
\hline $\begin{array}{c}\text { Compreensão das } \\
\text { questões }\end{array}$ & SIM & SIM & SIM & NÃO & SIM & $80 \%$ & $\begin{array}{c}\text { SEM } \\
\text { ALTERAÇÕES }\end{array}$ \\
\hline Clareza & SIM & SIM & SIM & SIM & SIM & $100 \%$ & $\begin{array}{c}\text { SEM } \\
\text { ALTERAÇÕES }\end{array}$ \\
\hline Coerência e Coesão & SIM & SIM & SIM & SIM & SIM & $100 \%$ & $\begin{array}{c}\text { SEM } \\
\text { ALTERAÇÕES }\end{array}$ \\
\hline Linguagem & SIM & SIM & SIM & NÃO & SIM & $80 \%$ & $\begin{array}{c}\text { SEM } \\
\text { ALTERAÇÕES }\end{array}$ \\
\hline $\begin{array}{l}\text { Distribuição das } \\
\text { questões }\end{array}$ & SIM & SIM & SIM & SIM & SIM & $100 \%$ & $\begin{array}{c}\text { SEM } \\
\text { ALTERAÇÕES }\end{array}$ \\
\hline $\begin{array}{c}\text { Itens de } \\
\text { Preenchimento }\end{array}$ & SIM & SIM & SIM & NÃO & SIM & $80 \%$ & $\begin{array}{c}\text { SEM } \\
\text { ALTERAÇÕES }\end{array}$ \\
\hline Aspecto visual & SIM & SIM & SIM & SIM & SIM & $100 \%$ & $\begin{array}{c}\text { SEM } \\
\text { ALTERAÇÕES }\end{array}$ \\
\hline $\begin{array}{c}\text { Adicionar alguma } \\
\text { questão }\end{array}$ & SIM & SIM & NÃO & NÃO & NÃO & $40 \%$ & ALTERAÇÕES \\
\hline $\begin{array}{l}\text { Propor mudança } \\
\text { estética }\end{array}$ & NÃO & SIM & NÃO & SIM & NÃO & $40 \%$ & ALTERAÇÕES \\
\hline
\end{tabular}

Fonte: Bispo et al. (2021)

Os quesitos passíveis de alterações são descritos juntamente com o comentário e/ou sugestão do especialista abaixo, empregando o valor de significância de $0,8 \%$ (Tabela 6). 
Tabela 6 - Modificações sugeridas pelos especialistas.

\begin{tabular}{|c|c|c|c|}
\hline Quesito & J1 & $\mathbf{J} 2$ & J4 \\
\hline $\begin{array}{c}\text { Adicionar alguma } \\
\text { questão }\end{array}$ & $\begin{array}{l}\text { Grau de escolaridade } \\
\text { da criança; Escola } \\
\text { privada ou pública; } \\
\text { Nível socioeconômico } \\
\text { dos pais. }\end{array}$ & $\begin{array}{l}\text { Percepção } \\
\text { pais/responsáveis em relação } \\
\text { ao tempo em que a criança } \\
\text { passa em frente às telas. }\end{array}$ & \\
\hline $\begin{array}{c}\text { Propor mudança } \\
\text { estética }\end{array}$ & & $\begin{array}{l}\text { Maior espaço para questões } \\
\text { abertas; Maior espaçamento } \\
\text { entre uma pergunta e outra; } \\
\text { Alinhar ao meio as frases } \\
\text { referentes a cada tópico; } \\
\text { Manter a mesma questão em } \\
\text { uma única página. }\end{array}$ & $\begin{array}{l}\text { Utilizar } \\
\text { Escala } \\
\text { Likert. }\end{array}$ \\
\hline
\end{tabular}

Fonte: Bispo et al. (2021).

\section{Etapa 2}

Após as sugestões serem coletadas, foi realizado um segundo contato com os juízes para revisar o instrumento. Dos cinco que participaram anteriormente, três responderam à etapa 2 do estudo. Na tabela 7 estão os itens que foram modificados de acordo com a avaliação dos experts.

Tabela 7. Modificações realizadas após revisão.

\begin{tabular}{cccc}
\hline & Questões a serem modificadas & \\
Juiz & Dados do Informante & $\begin{array}{c}\text { Uso de aparelhos } \\
\text { eletrônicos }\end{array}$ & $\begin{array}{c}\text { Marcos do } \\
\text { desenvolvimento }\end{array}$ \\
$\mathrm{J} 2.1$ & Nenhuma 11 & Nenhuma & 1,13 e 14 \\
$\mathrm{~J} 2.2$ & Nenhuma & 1 e 10 & Nenhuma \\
$\mathrm{J} 2.3$ & Nenhuma & Nenhuma \\
\hline
\end{tabular}

Fonte: Bispo et al. (2021)

O item 8 da seção "Dados do Informante" se refere à renda familiar. O juiz J2.1 sugeriu maior clareza nesta questão. Anteriormente a renda deveria ser informada por pessoa e isso dificultaria o entendimento do público, portanto, considerando a alteração sugerida, a resposta deverá ser a soma da renda de todos os que exercem atividade profissional no núcleo familiar.

A questão 11 diz respeito à faixa etária da criança, anteriormente com apenas 2 opções (2 anos a 3 anos e 11 meses ou 4 anos a 5 anos e 11 meses), e agora aberta para preenchimento com anos e meses.

Em relação aos itens 1, 13 e 14 da seção "Marcos do desenvolvimento", o juiz J2.1 sugeriu as seguintes modificações: item 1: revisão das categorias de faixa etária do início da produção de fala. Anteriormente estavam apenas as opções "1 ano; 2 anos; e 3 anos ou mais". A sugestão referiu-se a destrinchar melhor as idades, tornando as opções em "Entre 1 ano e 1 ano e 6 meses; Entre 1 ano e 6 meses e 2 anos; e 2 anos ou mais".

No item 13 foi sugerida uma definição mais clara de "Prática de atividades físicas". Após avaliação, a referência de "atividade física" foi alterada para "exercício físico" citando exemplos como: futebol, natação, artes marciais.

No item 14 foi solicitado que inseríssemos exemplos de atividades de lazer, e as escolhidas foram "ir ao parque, ir ao shopping...".

O juiz J2.3 sugeriu modificações em apenas 2 itens da seção "Uso de aparelho eletrônicos", sendo estes os itens 1 
e 10. A sugestão foi que acrescentássemos a opção "Computador de Mesa" nas alternativas referentes aos aparelhos que possuem na residência e qual o preferido da criança.

\section{Discussão}

A validade é um fator determinante na escolha e/ou aplicação de um instrumento de medida e é mensurada pela capacidade que ele tem de medir realmente aquilo que propõe (Bittencourt, 2011).

A validação de conteúdo é um método que não depende de dados estatísticos, pois resulta do julgamento de especialistas em determinada área do conhecimento (Correa, 2016).

$\mathrm{Na}$ área da saúde, o conceito de validade é descrito como sendo o grau no qual determinado instrumento é adequado para mensurar o que está para ser medido. A validação de conteúdo é passo essencial no desenvolvimento de novas medidas, porque representa o início de mecanismo para associar conceitos abstratos a indicadores observáveis e mensuráveis (De Morais et al., 2014). O julgamento dos juízes especialistas possibilita aperfeiçoar o instrumento com sugestões de mudanças no projeto piloto.

A validação do manual de orientação do projeto possibilitou a identificação de aspectos passíveis de aperfeiçoamento, dentro da legalidade e validade de aparência e conteúdo (De Souza et al., 2018). Corroborando com nosso estudo, relacionamos tais achados, identificando a grande importância da participação dos juízes nas etapas de validação e reconstrução de nosso instrumento para alcançarmos a versão mais adequada para a utilização, considerando a temática e o público-alvo.

Diversas recomendações para utilizar a internet com uso moderado e seguro, como fonte educativa e saudável, devem fazer parte da rotina dos atendimentos das crianças e de seus familiares (Correa, 2016).

A mediação dos pais é um elemento fundamental no uso das mídias, já que existem conteúdos que são inapropriados, e o tempo de uso irá depender da faixa etária da criança. Por outro lado, existem conteúdos adequados, interativos e educativos para cada idade e na quantidade de tempo correta (Grizólio \& Scorsolini-Comin, 2020).

Um exemplo é o sucesso na introdução das tecnologias educacionais nas escolas, uma vez que elas enriquecem a prática pedagógica e consequentemente melhoram os processos de aprendizagem (Correa, 2016).

Em 2016, a Sociedade Brasileira de Pediatria lançou o Manual de Orientações \#MenosTelas \#MaisSaúde com recomendações de tempo de uso de acordo com a idade sendo estes: "Evitar a exposição de crianças menores de 2 anos às telas, sem necessidade (nem passivamente!). Crianças com idades entre 2 e 5 anos, limitar o tempo de telas ao máximo de 1 hora/dia, sempre com supervisão de pais/cuidadores/ responsáveis. Crianças com idades entre 6 e 10 anos, limitar o tempo de telas ao máximo de 1-2 horas/dia, sempre com supervisão de pais/responsáveis. Adolescentes com idades entre 11 e 18 anos, limitar o tempo de telas e jogos de videogames a 2-3 horas/dia, e nunca deixar "virar a noite" jogando" (SBP, 2016).

Um estudo mostrou que $100 \%$ das crianças fazem uso de aparelhos audiovisuais, desses $50 \%$ tiveram o primeiro contato na faixa etária de idade de 8 a 12 meses, com o tempo médio de uso diário de 4 horas ou mais, seguido de 1 a 2 horas. A pesquisa demonstrou ainda que os pais são as primeiras pessoas a disponibilizarem os aparelhos, e este uso é feito em locais sem supervisão de um adulto mesmo que $87 \%$ relataram fazerem o controle do que seus filhos navegam na internet (Câmara, 2020).

Por já nascerem inseridos na cultura midiática e digital, é improvável e inviável que haja uma restrição do uso desses aparelhos nas crianças do mundo atual (Santana et al., 2021). O que cabe aos pais, educadores e profissionais de saúde que estão presentes nesta fase do desenvolvimento infantil onde os aspectos cognitivos, sociais e emocionais estão em construção, é mediar este uso, impor limites de tempo e gerenciar os conteúdos que suas crianças estão sendo expostas. É possível que 
através das tecnologias os processos de aprendizagem sejam potencializados e que estes recursos sejam utilizados de maneira benéfica (Nobre, 2021).

É importante ressaltar a importante associação entre o uso de dispositivos móveis e atrasos expressivos na fala. De acordo com estudos, um aumento de 30 minutos por dia no uso de dispositivos de mídia móvel foi associado a um risco 2 ou 3 vezes maior de atraso de fala expressivo relatado pelos pais (Van den Helvel, 2019). Uma revisão de 42 estudos, evidenciou que a maior quantidade de uso da tela foi negativamente associada à linguagem infantil, enquanto melhor qualidade do uso da tela, ou seja, programas educacionais e com cuidadores associaram-se positivamente às habilidades de linguagem infantil (Madigan, 2019).

O instrumento validado neste estudo poderá auxiliar familiares a compreender e refletir sobre os fatores envolvidos no tempo de uso de tela das crianças na primeira infância. Aos profissionais de saúde e educadores, este questionário poderá servir como um guia durante os atendimentos de avaliação e orientações a respeito da mediação do uso das telas.

Ampliar o conhecimento de responsáveis por crianças na primeira infância e profissionais que lidam com esse público a respeito dos prejuízos que o uso excessivo de telas pode trazer para o desenvolvimento global é de extrema importância no momento em que vivemos.

\section{Conclusão}

Com base na avaliação de fonoaudiólogos com experiência na área de linguagem infantil, o instrumento foi submetido às etapas de validação de conteúdo preconizadas em literatura e preenche os requisitos quanto à sua face e conteúdo.

O próximo passo do estudo compreende a aplicação do instrumento ao público-alvo em maior escala a fim de verificar as possibilidades de aplicabilidade e auxiliar em possíveis adequações que podem se fazer necessárias durante este processo.

Este estudo subsidiará o reconhecimento acerca do tempo de uso de tela na infância e suas implicações para o desenvolvimento global de crianças, podendo auxiliar no diagnóstico preciso deste tipo de situação, além de potencializar a criação de medidas preventivas e educacionais. Desta forma, indicamos a importância da continuidade de estudos com esta temática a fim de subsidiar o direcionamento frente ao uso de telas na infância.

\section{Referências}

Alexandre D. S. Alpes M. F; Reis A. C. M. B \& Mandrá P. P. (2020). Validation of a booklet on language developmental milestones in childhood. Rev. Cefac, $22(2), 1-14$.

Bittencourt H. R. (2011). Desenvolvimento e validação de um instrumento para avaliação de disciplinas na educação superior. Estudos em Avaliação Educacional,48 (22), 91-113.

Câmara H. V. (2020). Principais prejuízos biopsicossociais no uso abusivo da tecnologia na infância: percepções dos pais/Main biopsychosocial damages in abusive use of child technology: parental perceptions. Revista de Psicologia, 51 (14), 366-379.

Correa A. M. G. (2016). Impacto das tecnologias: o olhar dos pais acerca do viver saudável da criança. Revista de Enfermagem do Centro-Oeste Mineiro, 6 (1), 1915-1929.

Da Silva C. M., \& Ziegler M. M. (2016). Até que ponto os aparelhos eletrônicos ajudam e/ou atrapalham no desenvolvimento infantil? Uma Nova Pedagogia para a Sociedade Futura.

De Paula M. E., \& Pereira R. M. R. (2018) Os usos sociais que as crianças fazem das mídias na vida. Caderno de Educação, 49 (1), $105-122$.

Grizólio T. C., Scorsolini-Comin F. (2020). Como a mediação parental tem orientado o uso de internet do público infanto-juvenil? Psicologia Escolar e Educacional, 24 (1), 1-10.

Macarini S. M; Martins G. F; Minetto M. F \& Vieira M. L. (2010). Práticas parentais: uma revisão da literatura brasileira. Arquivos Brasileiros de Psicologia, 62(1), 119-134.

Madigan S. Association between screen time and children's performance on a developmental screening test. (2019). JAMA pediatrics, 173 (3): 244-250.

Maziero L. L; Ribeiro, D. F \& Reis H. M. (2016). Desenvolvimento Infantil e Tecnologia. Revista Interface Tecnológica, 13 (1), $13-23$. 
Research, Society and Development, v. 10, n. 17, e97101724357, 2021

(CC BY 4.0) | ISSN 2525-3409 | DOI: http://dx.doi.org/10.33448/rsd-v10i17.24357

Morais E. A. S; Rojas S. S. O \& Veiga V. C. (2014). Indicadores de saúde no cuidado ao paciente crítico neurológico. Rev Rene, 15 (2), 89-195.

Nobre J. N .P.(2021). Fatores determinantes no tempo de tela de crianças na primeira infância. Ciência \& Saúde Coletiva, 26 (3), $1127-1136$.

Pereira J. F. (2017) Influência dos fatores biológicos e socioeconômicos no desenvolvimento neuropsicomotor de pré-escolares. Saúde e Pesquisa, 10 (1), 135 144.

Santana M. I; Ruas M, A \& Queiroz P. H. B. (2021). O impacto do tempo de tela no crescimento e desenvolvimento infantil. Revista Saúde em Foco, 14 (1), $169-179$.

Silva A. F. (2016). Validation of an educational material as pedagogical tool on sexual initiation for teens, Journa of Nursing UFPE, 0 (1): 1-10.

Sociedade Brasileira de Pediatria. (2016). Saúde de Crianças e Adolescentes na era digital. Manual de Orientação n 1, $10 / 2016$.

Souza A. P; Zeigelboim B. S; Santos R. S. (2018). Atenção à disfagia orofaríngea no home care: gerenciamento fonoaudiológico: estudo de validação de aparência e conteúdo de um manual de orientação. Rev. Cefac, 20 (5): 640-647.

Ribeiro L. C. C. (2017). Construção e validação de manual sobre burnout em professores. Revista de Enfermagem do Centro-Oeste Mineiro, 7 (1), 13-17.

Van del Heuvel M. (2019). Mobile media device use is associated with expressive language delay in 18-month-old children. Journal of Developmental and Behavioral Pediatrics, 40 (2), 99-109.

World Health Organization. (2016). Guidelines on physical activity, sedentary behaviour and sleep for children under 5 years of age. World Health Organization. 